\title{
Textile Concentric Ring Electrodes: Influence of Position and Electrode Size on Cardiac Activity Monitoring
}

\author{
Gema Prats-Boluda $\left(\mathbb{D}^{1},{ }^{1}\right.$ Yiyao Ye-Lin, ${ }^{1}$ Francisco Pradas-Novella, ${ }^{1}$ \\ Eduardo Garcia-Breijo $\mathbb{D}^{2}{ }^{2}$ and Javier Garcia-Casado ${ }^{1}$ \\ ${ }^{1}$ Centro de Investigación e Innovación en Bioingeniería, Universitat Politècnica de València, Valencia 46022, Spain \\ ${ }^{2}$ Instituto Interuniversitario de Investigación de Reconocimiento Molecular y Desarrollo Tecnológico (IDM), Universitat Politècnica \\ de València, Valencia 46022, Spain
}

Correspondence should be addressed to Gema Prats-Boluda; gprats@ci2b.upv.es

Received 10 April 2018; Accepted 14 June 2018; Published 9 July 2018

Academic Editor: Mohammed T. Ghoneim

Copyright (C) 2018 Gema Prats-Boluda et al. This is an open access article distributed under the Creative Commons Attribution License, which permits unrestricted use, distribution, and reproduction in any medium, provided the original work is properly cited.

\begin{abstract}
Continuous ECG monitoring can play an important role in the rapid detection of pathological signatures and arrhythmias. Current systems use electrodes with limitations in wearable long-term applications and spatial selectivity. In this work, two sizes of textile concentric ring electrodes (TCRE42 and TCRE50) were developed and tested for monitoring cardiac activity. The low-cost devices were found to be easy to implement and to potentially have the advantages of textile electrodes for being lightweight, stretchable, adjustable, washable, and long-lasting. Both TCREs yielded similar signal detectability of different ECG waves. The optimal $P$ wave recording area (OPA) for detecting signals was on the upper right chest. Although the absolute signal amplitude of TCRE records was smaller than that of lead II, normalized amplitude in the OPA is similar for $T$ wave and higher for $P$ wave. The TCREs also allowed better analysis of $P$ wave morphology and were able to detect more right and left atrial depolarization waves. TCRE48 showed slightly better detectability, normalized amplitude, and spatial selectivity than TCRE50 within the OPA. The authors consider the TCRE suitable for use in ubiquitous mobile health care systems, especially for atrial activity monitoring and diagnosis.
\end{abstract}

\section{Introduction}

Measurement of electrophysiological signals is crucial for monitoring bodily states and facilitating clinical diagnoses. Of all the vital signs, the electrocardiogram (ECG) is the most important, since it is the primary diagnostic tool for cardiovascular diseases, the first cause of death in developed countries. The analysis of the different cardiac waves in the ECG signals is of major importance; the $P$ wave represents the depolarization wave that spreads from the sinoatrial node throughout the atria; the QRS complex represents ventricular depolarization, while the $T$ wave represents ventricular repolarization. The ECG provides information on heart rate and electrical conduction in the heart, which enables the diagnosis of a wide range of cardiac pathologies, such as sinus tachycardia or bradycardia, bundle branch block, AV blocks, or atrial/ventricular fibrillation, which can be dangerous or even cause sudden death [1].

Diagnosis is usually by analyzing 12-lead ECG shortterm records in clinics with benchtop equipment and wet disc electrodes. The most commonly used electrode for ECG monitoring is a gel-type silver/silver chloride $(\mathrm{Ag} / \mathrm{AgCl})$ electrode. However, as some pathologies require extended monitoring during the patient's normal activities, interest in mobile and long-term monitoring has grown for diagnosis, screening, risk assessment, prevention, and rehabilitation [2]. Wet electrodes are limited in their long-term use because they can irritate the skin. A conductive gel is a significant drawback as it can dry out over time, increasing contact impedance and losing signal quality [3]. Dry ECG electrodes have emerged as an alternative for long-term ECG monitoring. They do not need gel but operate through natural body 
moisture and perspiration [4]. The simplest dry electrode is a metal disc in direct contact with the skin, but its use is limited due to its stiffness and the fact that it can cause skin irritation. Dielectric materials between the electrode conductor and skin make it possible to use noncontact dry electrodes based on capacitive coupling. These are similar to dry contact electrodes and are also sensitive to motion artifacts but show better behavior in terms of less skin irritation [5]. One of the newest electrodes is made of conductive textile, with the advantage of being lightweight, flexible, stretchable, adjustable, washable, and long-lasting and does not cause skin irritation [6]. These electrodes are now becoming more popular because they are easy to use and cause minimal discomfort to the wearer. A number of proposals to embed these electrodes in normal clothing have produced promising results [7-9].

However, the standard 12-lead ECG also has limitations in diagnosing pathologies related to regions of anomalous local electrical activity, such as ventricular ischemia, atrial hypertrophies, or atrial flutter, requiring invasive electrophysiology, which involves significant risks for the patient and lengthens the diagnosis time [10]. This is due to the poor spatial resolution of conventional disc and rectangular electrodes, which are affected by the blurring effects of the different conductivities in the conductor. Spatial resolution is associated with the ability to differentiate the activity of dipole sources in different areas. Other configurations of electrode layouts and shapes are needed for better spatial resolution. To deal with this issue, modified electrode designs based on concentric ring electrodes (CREs) have been applied to several bioelectric signal recording and analysis, such as electrocardiographic (ECG) [11-15], electroenterographic (EEnG) [16], and electrohysterographic (EHG) signals [17]. The concentric ring design is a physical approximation to the Laplacian filter, which is the second spatial derivative of the measured potentials and essentially assigns more weight to changes in bioelectric dipoles under measurement points, to enable better differentiation between concurrent, closely spaced dipole sources [14]. CREs have been shown to be more effective at increasing spatial resolution than merely increasing the number of electrodes in an array, as in conventional body surface potential mapping [18]. This enhanced spatial resolution is of great interest in studying atrial activity, since there are fewer cardiac cells involved in atrial than ventricular activities. In fact, atrial fibrillation is the typical example of an arrhythmia in which a standard 12-lead ECG is insufficient to guide clinical management [19].

So far, CREs have been built on rigid substrates $[12,20$, 21] or flexible substrates such as polyester films [14, 16, 17] or polydimethylsiloxane (PDMS) elastomer [22]. We recently produced a CRE on a textile that can potentially benefit from the combination of long-term recording with high spatial resolution [23]. This initial experience showed it was possible to pick up cardiac activity with textile CRE (TCRE) and that silver inks performed better than PEDOT:PSS inks. The aim of the present work was to use this sensor to further explore the possibility of detecting and studying the different cardiac waves, with the emphasis on the $P$ wave, and to analyze the influence of the size and position of the TCRE on the results.

\section{Materials and Methods}

2.1. Textile Concentric Ring Electrodes (TCREs). Two sets of TCREs of two different sizes were designed, developed, and tested in the present work. The TCREs were integrated in elastic strips for adaptation to the body surface. Each strip had two TCREs of the same size with centers separated by $12 \mathrm{~cm}$. The TCREs were made up of an inner disc and an outer ring (Table 1). The TCRE's external diameter was similar to the distance between the surface of the chest and the heart (between 3.5 and $5.0 \mathrm{~cm}$ ) [19]. Compared to other CRE developed onto plastic substrates and used for picking up ECG signals in wet recordings (using electrolytic gel) [14], TCRE recording areas were increased in order to reduce the electrode impedance considering the signals recorded by TCRE under dry conditions (without electrolytic gel) [23].

Applying conductive patterns to textiles can be achieved by microcontact embedded electrodes, inkjet printing, and screen printing [24]. Conductive patterns of thin stainless steel, copper, or other metal wires are embroidered onto textile fabrics [25]. Screen printing is a popular method due to its relatively low cost and became possible by the development of polymer-based inks, which allow low curing temperatures compatible with textile substrates [26]. TCRE electrodes were thus produced using multilayer thick film serigraphic technology [23]. The screen for the conductors was a 230-mesh polyester material (PET 1500 90/230-48, Sefar, Thal, Switzerland), and the screen for the dielectric layer was a 175-mesh polyester material (PET 1500 68/17564 PW, Sefar). An UV film Dirasol 132 (Fujifilm, Tokyo, Japan) was used to transfer the stencil to the screen mesh. The final screen thickness was $10 \mu \mathrm{m}$ for the conductor screen and $15 \mu \mathrm{m}$ for the dielectric screen. The patterns were transferred to the screen by a UV light source. As for the materials: Mediatex TT ACQ $120 \mu \mathrm{m}$ textile (Junkers \& Muellers $\mathrm{GmbH}$, Mönchengladbach, Germany) was chosen for the substrate, C2131014D3 silver ink 59.75\% (Gwent Group, Pontypool, UK) as the conductive ink, and D2081009D6 polymer dielectric (Gwent Group, Pontypool, UK) as the dielectric ink. The electrical connection of the CREs with the measuring system was a snap fastener in each terminal of the electrodes, as shown in Figure 1. Further physical and electrical characterization, that is, final layer thickness, magnitude and phase of the impedance, and skin-electrode impedance, can be found at [23].

2.2. Recording Protocol. Nine recording sessions were performed on healthy male subjects aged between 21 and 43 years of age, with body mass indexes between 19.6 and $27.47 \mathrm{~kg} / \mathrm{m}^{2}$, thoracic contour between 81 and $119 \mathrm{~cm}$, and an internipple distance ranging from 19 to $29 \mathrm{~cm}$. The study was approved by the Universtitat Politècnica de València's Ethics Committee, and the Declaration of Helsinki was adhered to. The volunteers were previously informed of the nature of the study, briefed on the recording protocol, and signed a consent form.

The recording sessions were carried out with the subjects lying on a stretcher. They were asked to relax and 
TABle 1: Dimensions and distances of the TCREs.

\begin{tabular}{lcc}
\hline Parameter & TCRE50 & TCRE42 \\
\hline Inner disc diameter $(\mathrm{mm})$ & 16 & 16 \\
Ring internal diameter $(\mathrm{mm})$ & 36 & 28 \\
Ring external diameter $(\mathrm{mm})$ & 50 & 42 \\
Distance (between discs' centers) $(\mathrm{mm})$ & 120 & 120 \\
\hline
\end{tabular}

remain motionless to avoid fluctuations on the body surface electrocardiographic recordings due to changes in the heart position. Firstly, to reduce skin-contact impedance, the skin under the electrodes was previously exfoliated (Nuprep, Weaver and Company, USA) and shaved if necessary. Two disposable $\mathrm{Ag} / \mathrm{AgCl}$ electrodes (Kendall 100 Series Foam Electrodes, Medtronic, USA) were positioned on the left ankle and right wrist to obtain a standard lead II ECG signal, with a ground electrode on the right ankle.

To study the influence of the electrode position and to determine the best area to capture atrial activity, signals were recorded on a grid (see Figure 2) by two TCREs on elastic strips on the right of the subject vertically aligned with the right nipple, $8.5 \mathrm{~cm}$ above and $4.5 \mathrm{~cm}$ below it, respectively (see Figure 2). We adjusted the elastic strip so as to apply a light pressure to the dry TCRE and yield good electrode to skin contact. Subsequently, four bipolar concentric ECG signals (BC-ECG) (one per TCRE) and the standard lead II ECG signal were simultaneously recorded for 2 minutes with the subject at rest. The bands were then horizontally moved in steps of $2 \mathrm{~cm}$, until the entire area between the nipples was recorded, with between 6 and 8 recordings per subject per strip. The same scan was repeated twice more with downward vertical displacements of $2 \mathrm{~cm}$ each (see Figure 2). This process yielded between 72 and 96 recording points per subject and was performed for both electrode sizes (TCRE50 and TCRE42).

Commercial instrumentation amplifiers (Grass Technologies P511, AstroNova Inc., West Warwick, RI, USA) performed the signal conditioning of the four simultaneous BC-ECGs and lead II ECG signals. Signals were band-pass $(0.1-100 \mathrm{~Hz})$ and power line $(50 \mathrm{~Hz})$ filtered and then acquired at a $1000 \mathrm{~Hz}$ sampling frequency (USB NI-6229 BNC National Instrument acquisition card, Austin, TX).

2.3. ECG Analysis. The signals were digitally high-pass filtered with a linear-phase FIR filter with a cutoff frequency of $0.3 \mathrm{~Hz}$ to diminish baseline drift, and notch filter at
$100 \mathrm{~Hz}$ to attenuate power line harmonics. The first step in the ECG analysis was to obtain the ECG average beat for each recording position and subject; the Pan-Tompkins algorithm [27] was used for beat detection.

The electrocardiographic waves $(P, \mathrm{QRS}, T)$ were identified in the average beats. An experienced cardiologist determined if each wave was distinguishable (detected) or not. To determine the optimal recording area for picking up atrial activity with TCRE, the chest was divided into four areas, as shown in Figure 2. These were the upper and lower right areas (from the chest midline to the right above and below the nipple, resp.) and the upper and lower left areas (from the chest midline to the left above and below the nipple, resp.). Subsequently, the recording site percentage detectability (RSPD) for the different ECG waves $(P, Q, R, S$, and $T)$ in each area and the global recording area was worked out for each subject. The area that provided the highest mean $P$ wave detectability was selected as the optimal $P$ wave recording area (OPA).

Absolute and normalized peak-to-peak amplitude of cardiac waves at each recording site was also computed to further characterize and compare BC-ECGs. Median and interquartile values of absolute amplitude over all the recorded positions on each subject were calculated to obtain information on signal intensity and variability. The normalized amplitude is relevant since it provides information on the ability to analyze the morphology of each wave for clinical diagnosis. Parameters related to normalized amplitudes (or amplitude ratios) of the cardiac waves have been extensively used in the literature for different aims, ranging from biometric recognition systems to developing new diagnostic algorithms $[28,29]$. In the present work, peak-to-peak amplitudes of $P$ and $T$ waves were normalized to those of the whole average beat, that is, QRS in almost all cases. The median and maximum normalized amplitudes of the $P$ and $T$ waves were calculated over all the recorded positions on each subject. The absolute and normalized amplitudes of cardiac waves from lead II were also computed to compare the performance of TCREs with traditional cardiac sensing methods.

The capacity of TCRE to provide additional information on atrial activity to that from common disc electrodes was studied, especially the possibility of discriminating the electrical activity associated with the right and left atria in BCECG and lead II signals.

In order to assess the spatial selectivity of TCRE50 and TCRE42 in picking up cardiac activity, we used the $\mathrm{SS}_{\mathrm{w}}$ parameter. This was worked out for each cardiac wave as the average of the ratios of the absolute amplitude at one recording site and the four neighboring sites, as follows [12]:

$$
\mathrm{SS}_{\mathrm{w}}\left(x_{0}, y_{0}\right)=\frac{\left(\left(\mathrm{PP}\left(x_{0}, y_{0}\right) / \mathrm{PP}\left(x_{-1}, y_{0}\right)\right)+\left(\mathrm{PP}\left(x_{0}, y_{0}\right) / \mathrm{PP}\left(x_{+1}, y_{0}\right)\right)+\left(\mathrm{PP}\left(x_{0}, y_{0}\right) / \mathrm{PP}\left(x_{0}, y_{-1}\right)\right)+\left(\mathrm{PP}\left(x_{0}, y_{0}\right) / \mathrm{PP}\left(x_{0}, y_{+1}\right)\right)\right)}{4},
$$




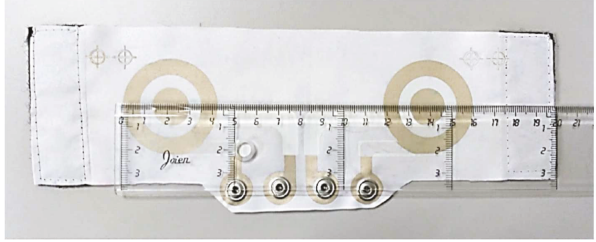

(a)

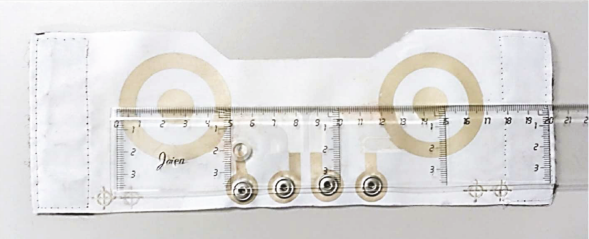

(b)

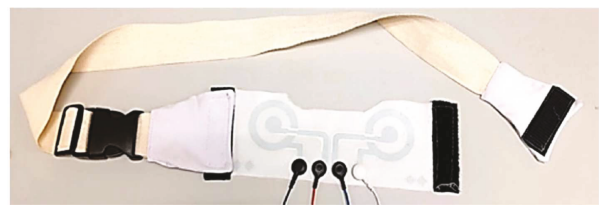

(c)

FIGURE 1: View of the elastic strip with silver concentric ring electrode TCRE42 (a) and silver concentric ring electrode TCRE50 (b). TCREs incorporated in an adjustable belt (c).

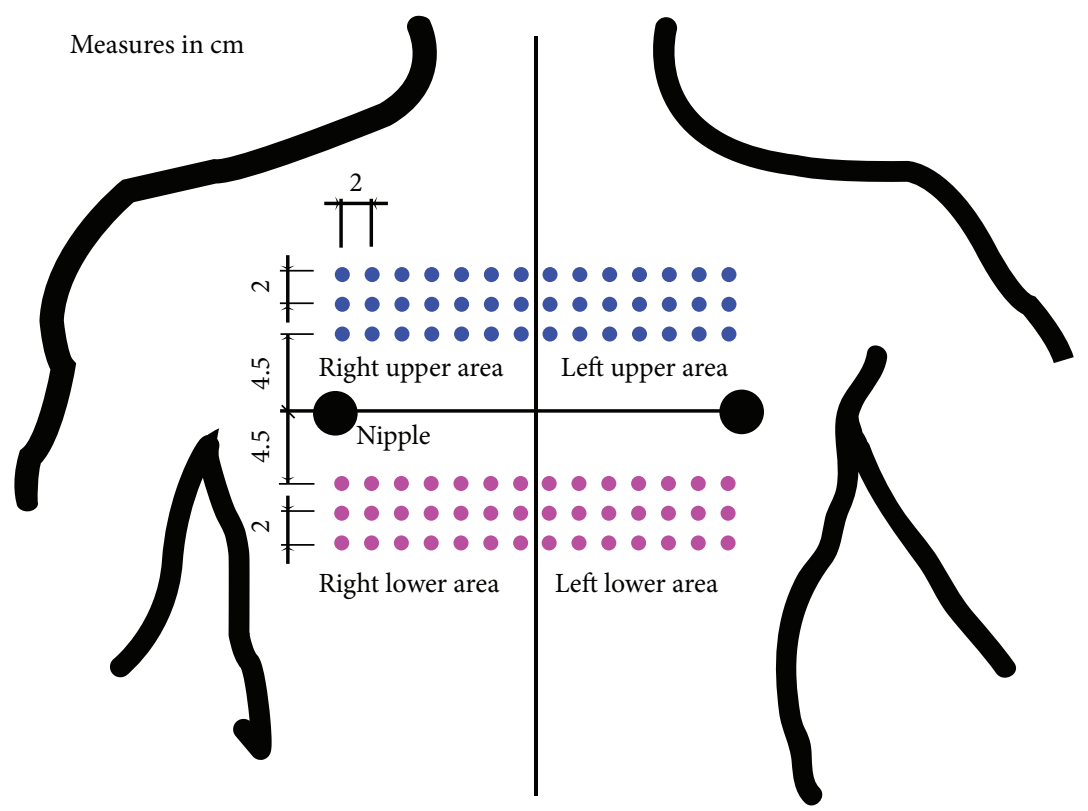

FIGURE 2: Grid of recording sites over the chest. Each dot represents a TCRE recording site. Horizontal and vertical distances between recording sites are indicated.

where PP is the peak-to-peak amplitude of the $P$, QRS, or $T$ waves in the average beat at location $\left(x_{0}, y_{0}\right)$ and its four adjacent average beats. Since the computation of this parameter requires records above and below the $\left(x_{0}, y_{0}\right)$, it was only computed for the central recording rows above and below the nipples (see Figure 2). This could not be computed for the first and last recording sites (columns) in this row. The average spatial selectivity was then computed for each subject.

\section{Results and Discussion}

Figure 3 shows the average beats of body surface BC-ECG mapping obtained from one subject using TCRE50, depicted with the same amplitude scale for all the recording positions. It can be seen that the maximum amplitude of the BC-ECG signals is usually found in the third and fourth rows, in the

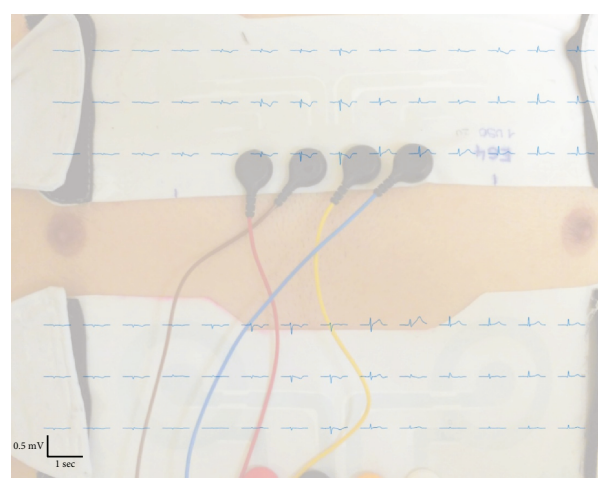

Figure 3: Body surface BC-ECG mapping obtained from subject number 1 using the TCRE50 electrode with the average beat at the different recording positions depicted in the same scale. 
recording positions from the median line to the left nipple (see Figure 3). The BC-ECG signals recorded at the first 5 points on the right in the sixth row tended to have a very low amplitude. This could be expected since the TCRE was quite far from the signal source and is in agreement with the findings of other authors [12]. The bipolar TCRE farfield rejection is proportional to the inverse fourth power of the distance between the signal wavefront and the electrode center [30]. Thus, the greater the distance from the ventricle, the greater the attenuation of the QRS complex. Nonetheless, the current signal conditioning and acquisition systems provide good quality signals for a wide range of input signal amplitudes such as those used in this study.

In fact, as clinical devices usually represent ECGs on a grid with adjustable vertical (and horizontal) scales for different traces, the use of autoscaled plots clinically makes sense. Figure 4 shows signals of Figure 3 with autoscaled vertical scales. It can be observed that, despite the low amplitude of the raw signal, both the QRS complex and $T$ wave can be easily identified in all the recording positions. This outperforms other systems that failed to detect ECG fiducial points ( $P$, QRS, $T$ waves) using PEDOT:PSS rectangularshaped electrodes dip-coated in polyester substrate [31]. The authors claim that this failure was due to the lower quantity of PEDOT:PSS absorbed by polyester and the high contact impedance between the skin and the polyester electrode [31]. In the present study, the screen-printed silver electrode probably provided better skin-electrode impedance, thus obtaining a high-quality ECG signal. The $P$ wave was only detected in the BC-ECG signal recorded at 38 of the 84 recording positions on this subject (shaded green in Figure 4), which are distributed in an area far removed from the ventricle. It seems that in these areas, the proportional attenuation of ventricular activity is higher than that of the atrial activity, resulting in an area of enhanced $P$ wave sensing, probably due to the relatively longer distance from the different cardiac signal sources.

The different ECG waves' RSPD of all the BC-ECG recording sites and each of the 4 predefined areas is shown in Table 2. It can be seen that TCRE50 and TCRE42 provided similar RSPD values for the different ECG waves. The QRS complex associated with ventricle depolarization can easily be recorded in any position, with $100 \%$ detectability for both TCREs. The $T$ wave associated with the repolarization of the ventricle was present in the ECG signal acquired at almost all the recording points and was identifiable in $97 \%$ of the cases. However, the $P$ wave associated with atrial depolarization was only detected in $41 \%$ of the recording sites by both TCREs. The detectability values of cardiac waves obtained in the present work are slightly higher than those obtained by other authors using CREs [14]. This may be related to various factors; firstly, the textile TCRE inherently provided better skin-electrode contact than CREs implemented on Melinex ST506 (plastic substrate) [14]. Furthermore, the adjustable belt used in the present study may also exert additional pressure and so may further improve skin-electrode contact, while the larger contact area of the TCRE poles than the CRE [14] may also contribute to better contact.

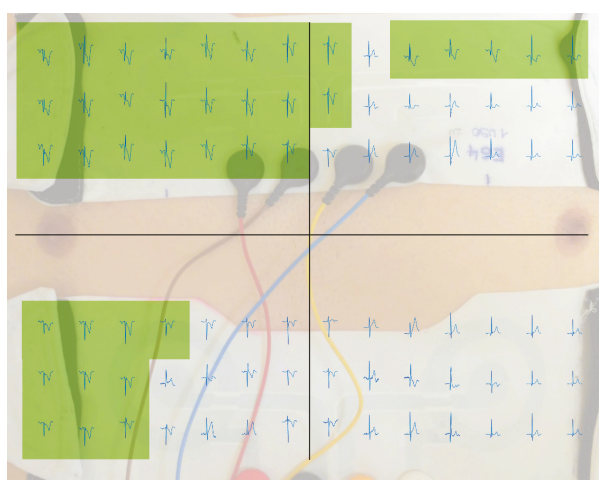

Figure 4: Body surface BC-ECG mapping obtained from subject number 1 using the TCRE50 electrode with the average beat at the different recording positions depicted in autoscale. The green areas are the recording sites in which the $P$ wave was detected in this subject.

TABLE 2: Mean and standard deviation of the recording site percentage detectability of the ECG waves of all the recording sites (global) and for the 4 predefined areas when using both TCREs.

\begin{tabular}{ccccccc}
\hline Electrode & Wave & $\begin{array}{c}\text { Global } \\
(\%)\end{array}$ & $\begin{array}{c}\text { Right } \\
\text { upper } \\
\text { area (\%) }\end{array}$ & $\begin{array}{c}\text { Left } \\
\text { upper } \\
\text { area } \\
(\%)\end{array}$ & $\begin{array}{c}\text { Right } \\
\text { lower } \\
\text { area (\%) }\end{array}$ & $\begin{array}{c}\text { Left } \\
\text { lower } \\
\text { area } \\
(\%)\end{array}$ \\
\hline TCRE50 & $P$ & $41 \pm 11$ & $86 \pm 14$ & $17 \pm 12$ & $52 \pm 29$ & $2 \pm 5$ \\
& QRS & $100 \pm 0$ & $100 \pm 0$ & $100 \pm 0$ & $100 \pm 0$ & $100 \pm 0$ \\
TCRE42 & QRS & $100 \pm 0$ & $100 \pm 0$ & $100 \pm 0$ & $100 \pm 0$ & $100 \pm 0$ \\
& $T$ & $96 \pm 3$ & $98 \pm 3$ & $98 \pm 4$ & $95 \pm 6$ & $96 \pm 6$ \\
\hline
\end{tabular}

No significant differences were found for the QRS complex and $T$ wave detectability between the ECG wave RSPD in the 4 predefined areas. However, $P$ wave detection varied widely in different areas. The RSPD results show a generalization of what was observed in Figure 3. Detectability on the left side was poor, almost null for the lower area and slightly better in the upper area. In the right lower area, the $P$ wave was detected in about $50 \%$ of the sites, with better performance in the right upper area $(86 \%$ and $88 \%$ for TCRE50 and TCRE42, resp.). This area was thus defined as the optimal $P$ wave recording area (OPA) for further analysis. These results agree with a previous study that found BC-ECG recorded at CMV1 had a superior performance at picking up atrial activity when comparing the signal detectability at four predetermined positions: CMV1 (position comparable to V1), CMV2, CMV4R, and CMV5 [14].

Table 3 shows the absolute and normalized amplitude statistics of the different ECG waves for both BC-ECG and lead II records. In general, the BC-ECG signal amplitude exhibited high variability, not only in the same position among the different subjects but also among the different recording points in the same subject (as can be seen in Figure 3). This latter can be seen in the interquartile range 
TABLE 3: Mean and standard deviation of the absolute signal amplitude statistics (median and interquartile range) and normalized amplitude statistics (median and maximum value) obtained using TCREs and lead II* $\left(P_{\mathrm{OPA}}\right.$ refers to the $P$ wave characteristics detected in the optimal $P$ wave recording area).

\begin{tabular}{|c|c|c|c|c|c|}
\hline \multirow[b]{2}{*}{ Electrode } & \multirow[b]{2}{*}{ Wave } & \multicolumn{2}{|c|}{ Absolute amplitude } & \multicolumn{2}{|c|}{ Normalized amplitude } \\
\hline & & Median $(\mu \mathrm{V})$ & Interquartile range $(\mu \mathrm{V})$ & Median & Maximum \\
\hline \multirow{4}{*}{ TCRE50 } & $P$ & $11.2 \pm 2.9$ & $14.7 \pm 8.8$ & $0.09 \pm 0.03$ & $0.29 \pm 0.13$ \\
\hline & $P_{\mathrm{OPA}}$ & $11.5 \pm 4.9$ & $10.2 \pm 6.2$ & $0.14 \pm 0.05$ & $0.26 \pm 0.11$ \\
\hline & QRS & $158.2 \pm 38.8$ & $296.3 \pm 193.5$ & - & - \\
\hline & $T$ & $38.7 \pm 11.0$ & $75.4 \pm 47.4$ & $0.28 \pm 0.06$ & $0.76 \pm 0.14$ \\
\hline \multirow{4}{*}{ TCRE42 } & $P$ & $8.1 \pm 2.1$ & $9.9 \pm 5.8$ & $0.09 \pm 0.03$ & $0.32 \pm 0.19$ \\
\hline & $P_{\mathrm{OPA}}$ & $8.8 \pm 3.3$ & $7.9 \pm 3.1$ & $0.16 \pm 0.07$ & $0.32 \pm 0.19$ \\
\hline & QRS & $118.2 \pm 32.6$ & $190.3 \pm 87.9$ & - & - \\
\hline & $T$ & $30.6 \pm 8.3$ & $59.9 \pm 37.1$ & $0.30 \pm 0.07$ & $0.89 \pm 0.22$ \\
\hline \multirow{3}{*}{ Lead II } & $P$ & $116.0 \pm 29.2$ & - & $0.12 \pm 0.09$ & - \\
\hline & QRS & $1182.9 \pm 467.1$ & - & - & - \\
\hline & $T$ & $316.3 \pm 139.2$ & - & $0.28 \pm 0.14$ & - \\
\hline
\end{tabular}

*The constant location of lead II electrodes means some statistics have no practical meaning; these are indicated with dashes to avoid misinterpretation. Similarly, a double dash is used for the normalized QRS amplitude statistic, which is always 1.

value of the amplitude, which was of the same order of magnitude or even greater than the median value. One cause of the high variability in the BC-ECG signal amplitude can be differences in the pressure exerted by the elastic strip used to assure good electrode-skin contact [32]. In future studies, we intend to carry out measures to control this factor. This has been explored in other works in the literature either with direct measures [33] or through the use of inflatable vests/ cuffs in which the electrodes are embedded, allowing the precise control of the pressure exerted [25]. Nevertheless, the signal amplitude acquired using both TCREs was typically much smaller than that of lead II for all the ECG waves. This was to be expected, since the distance between the outer ring and the central disc was much shorter than the distance across the body between the lead II electrodes. This result agrees with other authors who simultaneously recorded a standard 12-lead ECG signal and BC-ECG signals using CRE $[15,18,12]$. TCRE50 recorded a higher median signal amplitude than TCRE42, confirming that signal amplitude is dependent on interpole distance. The median amplitude of different ECG waves obtained from the TCREs was similar to that obtained by other authors that used CRE with outer diameters ranging from 21.6 to $45.6 \mathrm{~mm}$ [15]. As expected, the $P$ wave amplitude acquired using both TCREs was very low (median value $\sim 11 \mu \mathrm{V}$ ), which can be a challenge for the signal conditioning system. In the optimal recording area, the median value of $P$ wave amplitude was slightly higher, with a lower interquartile range than that obtained from all the tested recording points.

As for the normalized $P$ and $T$ wave amplitudes computed from all the recording positions, TCREs generally provide similar values to those of standard lead II, while the maximum normalized BC-ECG amplitudes are at least twice as large as those of lead II. This suggests that this ratio, and thus the "contrast" with which the cardiac waves can be observed, is highly dependent on the TCRE recording position. In the $P$ wave optimal recording area $\left(P_{\mathrm{OPA}}\right)$, the median normalized amplitude value was almost double that of all the recording sites and slightly higher than that of lead II. This agrees with other authors who compared the normalized amplitude of BC-ECG signals acquired by CRE and standard 12-lead ECG recording and obtained the highest normalized amplitude at position V1 [14]. Wang et al. also found that signals obtained by CRE maintain good contrast for identifying $P$ waves associated with atrial activity, the normalized $P$ wave amplitude being $13 \%$ for lead I and $18 \%$ for the BC-ECG signal acquired using CRE at V1 [22]. Regarding the influence of electrode size, TCRE42 provided higher median and maximum normalized amplitude for both the $P$ and $T$ wave than TCRE50, which suggests that it is easier to identify and assess the morphology of these cardiac waves with the smaller electrode.

Regarding the possibility of discriminating the electrical activity associated with the right and left atria in BC-ECG and lead II signals, the associated P1 and P2 cardiac waves were identified in all the subjects by both TCRE50 and TCRE42, although they were observed in only 6 of the 9 subjects in standard lead II (see Figure 5). This suggests that both TCREs offered superior performance than standard lead II in the identification of local activity, which could be of great importance in the diagnosis of pathologies such as atrial hypertrophies or atrial flutter [10].

The average spatial selectivity computed from individual subjects ranged from 0.75 to 3.07 , which was of the same order of magnitude as that obtained by Besio and Chen [12]. The mean and standard deviation of the average spatial selectivity for each TCRE size and cardiac wave are shown in Table 4. Similar spatial selectivity values were obtained for different ECG waves. In general, TCRE42 offers better spatial selectivity than TCRE50, regardless of the ECG wave. This 


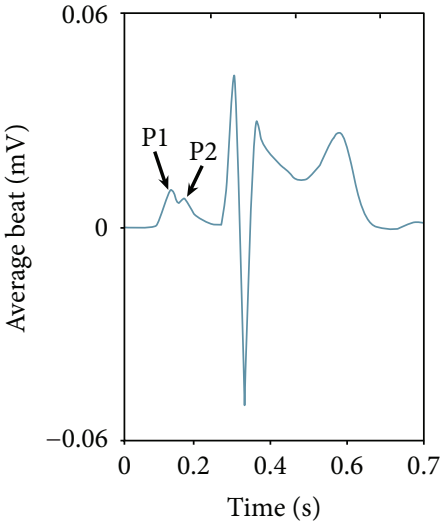

(a)

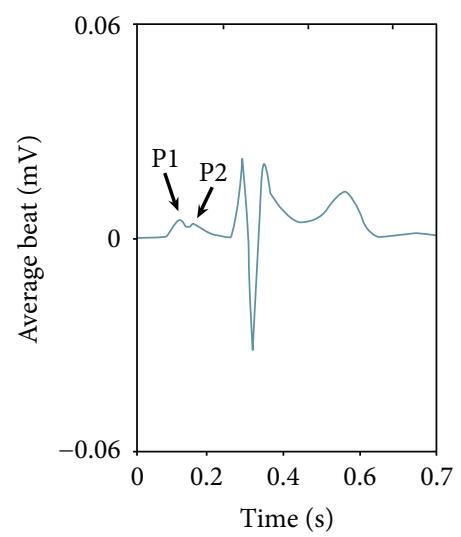

(b)

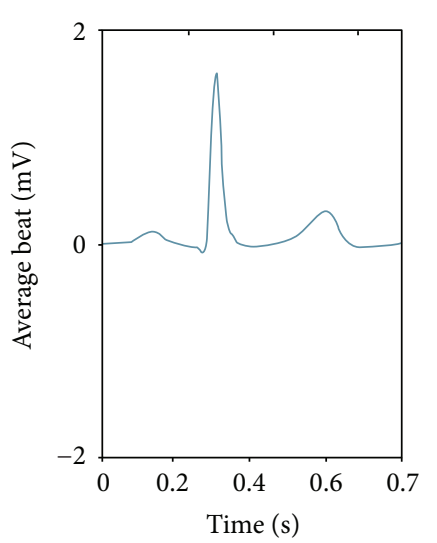

(c)

FIgURE 5: Average beats obtained from subject number 2. (a) Using TCRE50 in the OPA; (b) using TCRE42 in the OPA; (c) lead II.

TABLE 4: Mean and standard deviation of TCREs' average spatial selectivity for the different ECG waves.

\begin{tabular}{lcc}
\hline & TCRE50 & TCRE42 \\
\hline$P$ & $1.39 \pm 0.27$ & $1.74 \pm 0.53$ \\
QRS & $1.49 \pm 0.32$ & $1.84 \pm 0.38$ \\
$T$ & $1.68 \pm 0.47$ & $1.91 \pm 0.74$ \\
\hline
\end{tabular}

was as expected, since the bigger the electrode, the wider the recording area, thus the more the mutual information between adjacent recording points and the less the signal amplitude variability between them.

The results obtained suggested that the TCRE is suitable for high spatial resolution ECG long-term recordings in a comfortable manner. However, the study is not exempt from limitations; firstly, relatively few subjects were involved, which means the results should be further corroborated by a larger database. A study should also be carried out on TCRE biocompatibility, long-term wearability, reusability after cleaning, sensitivity to perspiration, motion artifact immunity, and cytotoxicity so as to adapt TCREs for mobile healthcare applications [34].

Other limitation of the present study is that it has been performed exclusively in male volunteers. Results derived from the study could be different in the case of women. Gender-dependent differences in breast tissue and in electrical substrate of the human heart could provoke different results $[35,36]$. Indeed, slight differences were found between healthy males and females in the limb-lead amplitudes $(P, Q, R, S$, and $T)$. QRS and $P-R$ duration was significantly shorter in women, and amplitudes $(R, S$, and $T)$ in precordial leads were significantly smaller [37]. The BMI of the subjects could also affect the measurements [38], as well as the age $[39,40]$. In the present study, ECG recordings were carried out in men volunteers with normal BMI and in a fairly narrow age range (young adults). In future works, we would deal to overcome this limitation (gender, BMI, and ECG) analyzing how ECG signals recorded with CRE are influenced by these issues and checking if the optimal area for $P$ wave recording is modified.

\section{Conclusions}

This paper describes two new textile concentric ring electrodes (TCRE42 and TCRE50) and the results of tests on their ability to sense cardiac activity as compared to standard lead II. The proposed TCREs are easy to implement, can be produced at little cost, and potentially have the advantages of textile electrodes in terms of lightness, flexibility, adjustability, washability, and long life.

Similar signal detectability was obtained by both TCREs for different ECG waves. Based on $P$ wave detectability in BC-ECG recordings, the optimal $P$ wave recording area (OPA) was found to be on the right upper chest. Although the absolute signal amplitude of BC-ECG was smaller than that of lead II, BC-ECG presented a similar normalized $T$ wave amplitude and even higher $P$ wave amplitude in the OPA, allowing better analysis of its morphology. The right and left atrial depolarization waves (P1 and P2) could also be observed in more subjects in the TCRE recordings than in the standard lead II ECG signals. The smaller electrode (TCRE42) yielded slightly better detectability results, normalized amplitude, and spatial selectivity than the TCRE50 in the OPA.

The authors consider that the proposed TCRE could be used in the near future in ubiquitous mobile health care systems, especially for atrial activity monitoring and diagnosis.

\section{Data Availability}

The data used to support the findings of this study are available on request from the corresponding author.

\section{Conflicts of Interest}

The authors declare that there is no conflict of interest regarding the publication of this paper.

\section{Acknowledgments}

This work was supported by grants from the Ministerio de Economía y Competitividad and the European Regional 
Development Fund (DPI2015-68397-R) (MINECO/FEDER). This work was also supported by the Spanish Government/ FEDER funds (MAT2015-64139-C4-3-R) (MINECO/FEDER).

\section{References}

[1] J. Malmivuo and R. Plonsey, "The basis of ECG diagnosis," in Bioelectromagnetism: Principles and Applications of Bioelectric and Biomagnetic Fields, pp. 320-335, Oxford University Press, 1995.

[2] A. Cömert, M. Honkala, and J. Hyttinen, "Effect of pressure and padding on motion artifact of textile electrodes," Biomedical Engineering Online, vol. 12, no. 1, p. 26, 2013.

[3] A. Searle and L. Kirkup, "A direct comparison of wet, dry and insulating bioelectric recording electrodes," Physiological Measurement, vol. 21, no. 2, pp. 271-283, 2000.

[4] J. G. Webster and J. W. Clark, Medical Instrumentation: Application and Design, John Wiley \& Sons, 2010.

[5] E. Nemati, M. Deen, and T. Mondal, "A wireless wearable ECG sensor for long-term applications," IEEE Communications Magazine, vol. 50, no. 1, pp. 36-43, 2012.

[6] W. Zeng, L. Shu, Q. Li, S. Chen, F. Wang, and X.-M. Tao, "Fiber-based wearable electronics: a review of materials, fabrication, devices, and applications," Advanced Materials, vol. 26, no. 31, pp. 5310-5336, 2014.

[7] R. Paradiso, G. Loriga, and N. Taccini, "A wearable health care system based on knitted integrated sensors," IEEE Transactions on Information Technology in Biomedicine, vol. 9, no. 3, pp. 337-344, 2005.

[8] M. Catrysse, R. Puers, C. Hertleer, L. Van Langenhove, H. van Egmond, and D. Matthys, "Towards the integration of textile sensors in a wireless monitoring suit," Sensors and Actuators A: Physical, vol. 114, no. 2-3, pp. 302-311, 2004.

[9] A. Vehkaoja, J. Verho, A. Cömert, M. Honkala, and J. Lekkala, Wearable System for EKG Monitoring - Evaluation of NightTime Performance, Springer, Berlin, Heidelberg, 2012.

[10] Y. Wang, P. S. Cuculich, J. Zhang et al., "Noninvasive electroanatomic mapping of human ventricular arrhythmias with electrocardiographic imaging," Science Translational Medicine, vol. 3, no. 98, p. 98ra84, 2011.

[11] O. Makeyev and W. Besio, "Improving the accuracy of Laplacian estimation with novel variable inter-ring distances concentric ring electrodes," Sensors, vol. 16, no. 6, p. 858, 2016.

[12] W. Besio and T. Chen, "Tripolar Laplacian electrocardiogram and moment of activation isochronal mapping," Physiological Measurement, vol. 28, no. 5, pp. 515-529, 2007.

[13] B. He and R. J. Cohen, "Body surface Laplacian ECG mapping," IEEE Transactions on Biomedical Engineering, vol. 39, no. 11, pp. 1179-1191, 1992.

[14] G. Prats-Boluda, Y. Ye-Lin, J. M. Bueno-Barrachina, R. Rodriguez De Sanabria, and J. Garcia-Casado, "Towards the clinical use of concentric electrodes in ECG recordings: influence of ring dimensions and electrode position," Measurement Science and Technology, vol. 27, no. 2, article 025705, 2016.

[15] Y. Ye-Lin, J. M. Bueno-Barrachina, G. Prats-boluda, R. Rodriguez de Sanabria, and J. Garcia-Casado, "Wireless sensor node for non-invasive high precision electrocardiographic signal acquisition based on a multi-ring electrode," Measurement, vol. 97, pp. 195-202, 2017.
[16] V. Zena-Giménez, J. Garcia-Casado, Y. Ye-Lin, E. GarciaBreijo, and G. Prats-Boluda, "A flexible multiring concentric electrode for non-invasive identification of intestinal slow waves," Sensors, vol. 18, no. 2, p. 396, 2018.

[17] Y. Ye-Lin, J. Alberola-Rubio, G. Prats-boluda, A. Perales, D. Desantes, and J. Garcia-Casado, "Feasibility and analysis of bipolar concentric recording of electrohysterogram with flexible active electrode," Annals of Biomedical Engineering, vol. 43, no. 4, pp. 968-976, 2015.

[18] M. Rodrigo, A. M. Climent, A. Liberos et al., "Minimal configuration of body surface potential mapping for discrimination of left versus right dominant frequencies during atrial fibrillation," Pacing and Clinical Electrophysiology, vol. 40, no. 8, pp. 940-946, 2017.

[19] S. Mittal, C. Movsowitz, and J. S. Steinberg, "Ambulatory external electrocardiographic monitoring," Journal of the American College of Cardiology, vol. 58, no. 17, pp. 17411749, 2011.

[20] C. C. Lu and P. P. Tarjan, "An ultra-high common-mode rejection ratio (CMRR) AC instrumentation amplifier for Laplacian electrocardiographic measurement," Biomedical Instrumentation \& Technology, vol. 33, no. 1, pp. 76-83, 1999.

[21] G. Prats-Boluda, J. Garcia-Casado, J. L. Martinez-de-Juan, and Y. Ye-Lin, "Active concentric ring electrode for non-invasive detection of intestinal myoelectric signals," Medical Engineering \& Physics, vol. 33, no. 4, pp. 446-455, 2011.

[22] K. Wang, U. Parekh, T. Pailla, H. Garudadri, V. Gilja, and T. N. Ng, "Stretchable dry electrodes with concentric ring geometry for enhancing spatial resolution in electrophysiology," Advanced HealthCare Materials, vol. 6, no. 19, 2017.

[23] J. Lidón-Roger, G. Prats-Boluda, Y. Ye-Lin, J. Garcia-Casado, and E. Garcia-Breijo, "Textile concentric ring electrodes for ECG recording based on screen-printing technology," Sensors, vol. 18, no. 1, p. 300, 2018.

[24] M. P. Aleksandrova, G. H. Dobrikov, and G. D. Kolev, "Electrical characterization of PEDOT:PSS based flexible organic optoelectronic devices," International Journal on "Technical and Physical Problems of Engineering”, vol. 8, no. 26, pp. 7176, 2016.

[25] B. Taji, S. Shirmohammadi, V. Groza, and I. Batkin, "Impact of skin-electrode interface on electrocardiogram measurements using conductive textile electrodes," IEEE Transactions on Instrumentation and Measurement, vol. 63, no. 6, pp. 14121422, 2014.

[26] E. Garcia-Breijo, G. Prats-Boluda, J. V. Lidon-Roger, Y. Ye-Lin, and J. Garcia-Casado, "A comparative analysis of printing techniques by using an active concentric ring electrode for bioelectrical recording," MicroElectronics International, vol. 32, no. 2, pp. 103-107, 2015.

[27] J. Pan and W. J. Tompkins, "A real-time QRS detection algorithm," IEEE Transactions on Biomedical Engineering, vol. BME-32, no. 3, pp. 230-236, 1985.

[28] S. Sovilj, A. Van Oosterom, G. Rajsman, and R. Magjarevic, "ECG-based prediction of atrial fibrillation development following coronary artery bypass grafting," Physiological Measurement, vol. 31, no. 5, pp. 663-677, 2010.

[29] A. Fratini, M. Sansone, P. Bifulco, and M. Cesarelli, "Individual identification via electrocardiogram analysis," Biomedical Engineering Online, vol. 14, no. 1, p. 78, 2015.

[30] C. C. Lu and P. P. Tarjan, "Pasteless, active, concentric ring sensors for directly obtained Laplacian cardiac electrograms," 
Journal of Medical and Biological Engineering, vol. 22, pp. 199203, 2002.

[31] A. Ankhili, X. Tao, C. Cochrane, D. Coulon, and V. Koncar, "Washable and reliable textile electrodes embedded into underwear fabric for electrocardiography (ECG) monitoring," Materials, vol. 11, no. 2, p. 256, 2018.

[32] B. Taji, A. D. C. Chan, and S. Shirmohammadi, "Effect of pressure on skin-electrode impedance in wearable biomedical measurement devices," IEEE Transactions on Instrumentation and Measurement, pp. 1-13, 2018.

[33] N. Meziane, S. Yang, M. Shokoueinejad, J. G. Webster, M. Attari, and H. Eren, "Simultaneous comparison of 1 gel with 4 dry electrode types for electrocardiography," Physiological Measurement, vol. 36, no. 3, pp. 513-529, 2015.

[34] H.-C. Jung, J.-H. Moon, D.-H. Baek et al., "CNT/PDMS composite flexible dry electrodesfor long-term ECG monitoring," IEEE Transactions on Biomedical Engineering, vol. 59, no. 5, pp. 1472-1479, 2012.

[35] G. Salama and G. C. L. Bett, "Sex differences in the mechanisms underlying long QT syndrome," American Journal of Physiology-Heart and Circulatory Physiology, vol. 307, no. 5, pp. H640-H648, 2014.

[36] A. J. Moss, "Gender differences in ECG parameters and their clinical implications," Annals of Noninvasive Electrocardiology, vol. 15, no. 1, pp. 1-2, 2010.

[37] E. Simonson, H. Blackburn, T. C. Puchner, P. Eisenberg, F. Ribeiro, and M. Meja, "Sex differences in the electrocardiogram," Circulation, vol. 22, no. 4, pp. 598-601, 1960.

[38] S. Kurisu, H. Ikenaga, N. Watanabe et al., "Electrocardiographic characteristics in the underweight and obese in accordance with the World Health Organization classification," IJC Metabolic \& Endocrine, vol. 9, pp. 61-65, 2015.

[39] J. Vicente, L. Johannesen, L. Galeotti, and D. G. Strauss, "Mechanisms of sex and age differences in ventricular repolarization in humans," American Heart Journal, vol. 168, no. 5, pp. 749-756.e3, 2014.

[40] J. Xue and R. M. Farrell, "How can computerized interpretation algorithms adapt to gender/age differences in ECG measurements?," Journal of Electrocardiology, vol. 47, no. 6, pp. 849-855, 2014. 


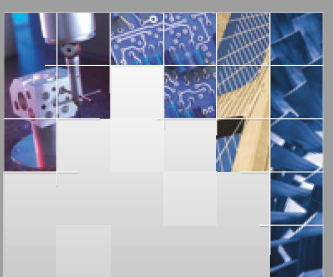

\section{Enfincering}
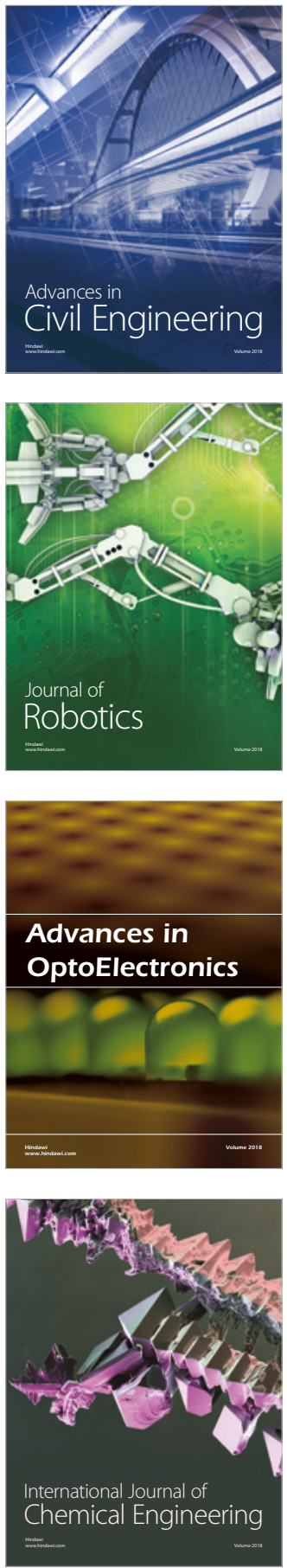

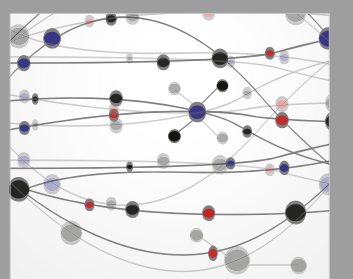

\section{Rotating \\ Machinery}

The Scientific World Journal

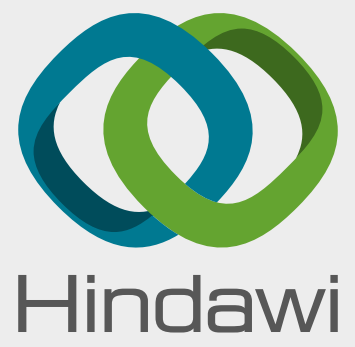

Submit your manuscripts at

www.hindawi.com
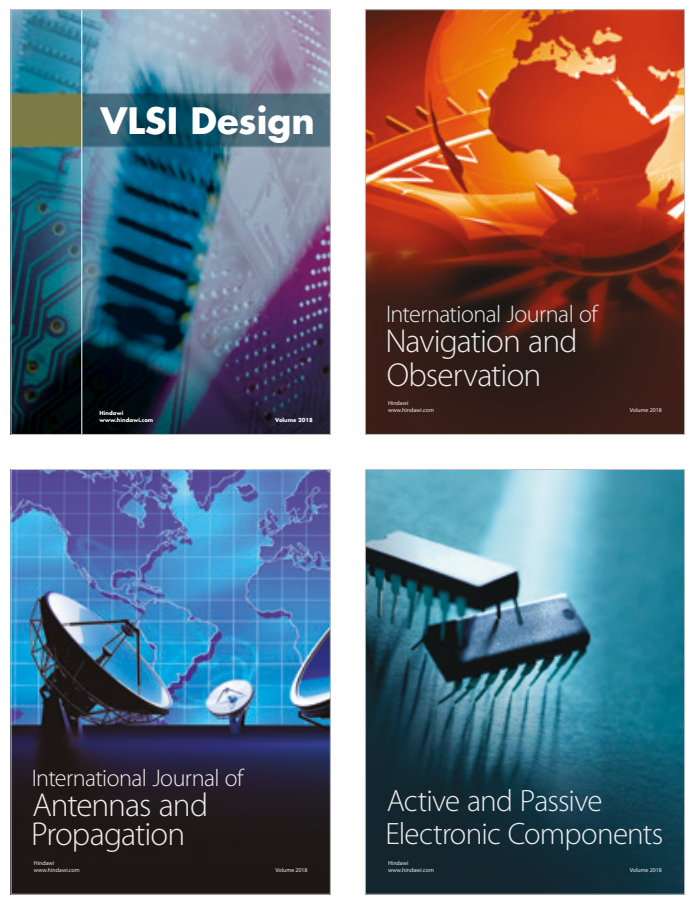
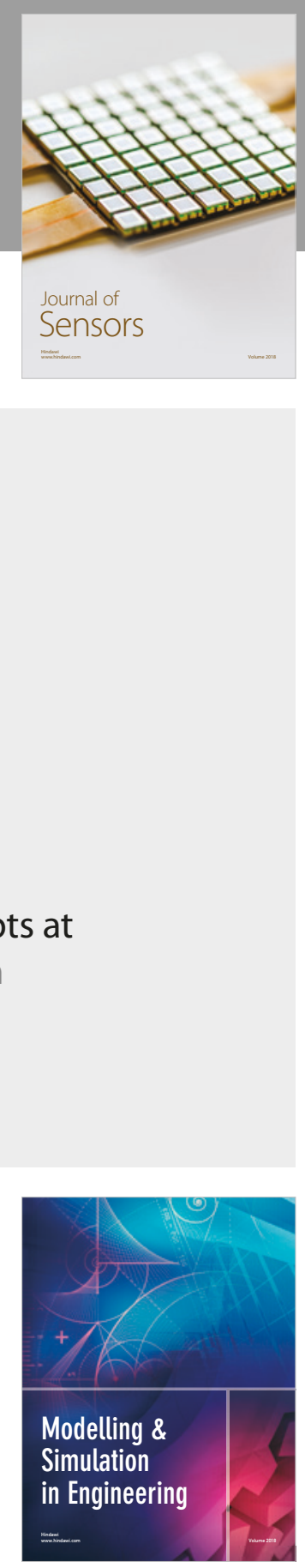

\section{Advances \\ Multimedia}
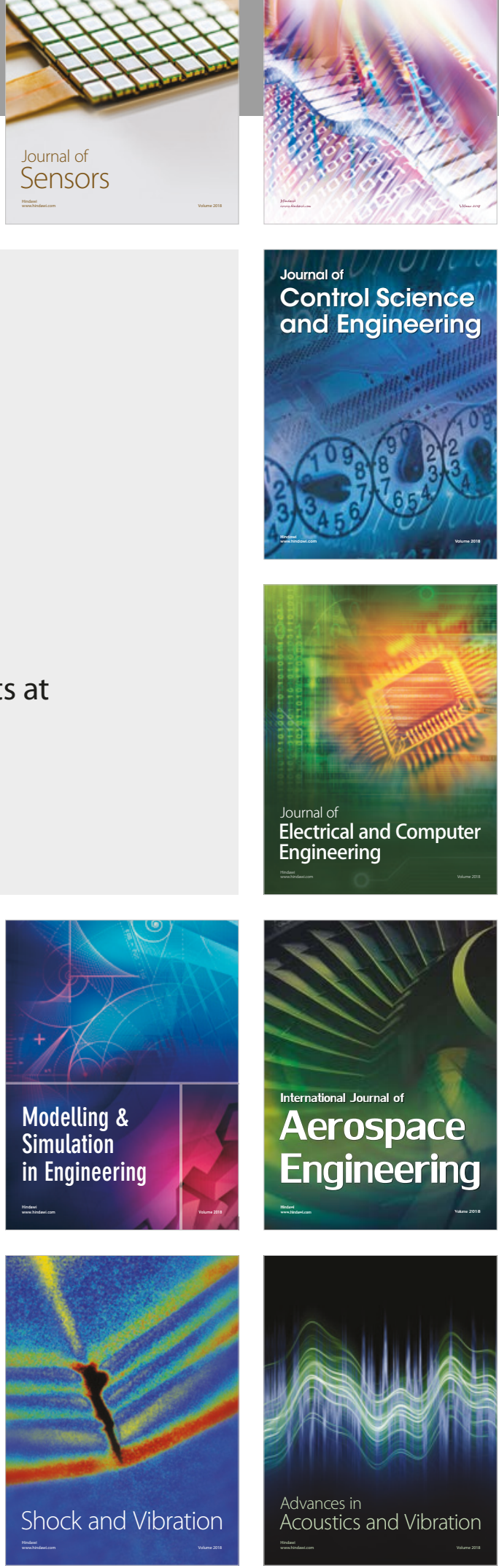